\title{
¿Qué es el absolutismo lógico? Respuesta a Alejandro Tomasini
}

\author{
Víctor MANuel Hernández MárqueZ \\ Dirección de Difusión Cultural \\ Universidad Autónoma Chapingo \\ vmarquez@taurus1.chapingo.mx
}

\begin{abstract}
Resumen: El presente texto es una respuesta a la reseña que el profesor Tomasini ha hecho de mi libro Lógica, lenguaje y realidad. Argumento que su crítica está equivocada en al menos cinco puntos importantes: 1) Descuida las diferencias entre la distinción de van Heijenoort y la de Hintikka, entre la lógica como lenguaje y el lenguaje como medio universal; y de paso, también la mía. 2) Su demanda por una definición clara de la noción de absolutismo lógico se encuentra desencaminada. 3) Confunde mi interpretación del absolutismo de Wittgenstein con la llamada teoría pictórica. 4) La interpretación heredada sobre la aritmética de Wittgenstein, sostenida por Tomasini, es errónea en varios sentidos y no puede usarse contra la interpretación que él opone; esto es, contra el logicismo. 5) Por último, la discusión de Tomasini sobre la noción de analiticidad de Frege se encuentra simplemente fuera de lugar.
\end{abstract}

Palabras clave: logicismo, teoría pictórica, lógica como lenguaje, definiciones

En el número 49 de Diánoia, el doctor Tomasini ha publicado una reseña de mi libro Lógica, lenguaje y realidad (Universidad Autónoma de Chihuahua, Chihuahua, 2001); con ello me ha hecho un gran honor y al mismo tiempo me ha dado la oportunidad de volver a reflexionar sobre algunos aspectos de mi trabajo que pueden despertar ciertos malentendidos, además de que me permite profundizar en aquellos puntos en los cuales existe una clara divergencia entre nosotros.

Para empezar, el autor ubica mi texto como un trabajo "básicamente" de historia de la lógica, aunque de inmediato rectifica al señalar que mi propósito apunta a describir y discutir la concepción absolutista o universalista de la lógica. En consecuencia, el libro no es una historia de la lógica, sino que se ocupa de filosofía de la lógica, y, en particular, de una doctrina muy peculiar que podemos encontrar en la obra de Leibniz y Frege, pero también en los escritos de Russell y Wittgenstein.

Ahora bien, ¿en qué consiste dicha concepción? El profesor Tomasini afirma que soy incapaz de ofrecer una respuesta clara y unívoca a semejante pregunta (p. 184), y luego enumera nueve observaciones mías, fuera de contexto o incompletas, sobre el absolutismo lógico, y añade que "es prácticamente todo" (p. 185) lo que el lector podrá encontrar sobre la misma. Daré sólo un par de ejemplos ilustrativos de lo poco afortunado que ha sido al citarme. 
Según él, afirmo de manera categórica que:

d) "el absolutismo lógico no es una teoría" (p. 184)

cuando en realidad mi texto dice: "como resaltó van Heijenoort, el absolutismo lógico no es una teoría abiertamente formulada y defendida, pero sus características generales pueden encontrarse, por ejemplo, en el uso de los cuantificadores por parte de Frege y Russell, en la manera como Frege entiende las reglas de inferencia [etcétera]". Desde luego, tratándose de una paráfrasis, Tomasini no me desfigura a mí, sino a van Heijenoort, ${ }^{1}$ pero eso es lo de menos, porque lo lamentable es que, al citarme así, el autor se priva de ofrecer al lector un rasgo importante de la concepción absolutista. Sin embargo, lo más extraño del asunto es que más adelante emplea (p. 189) el mismo rasgo para arrojar dudas sobre la tesis general de mi libro:

Nótese también que él mismo admite en relación con el absolutismo que "en Frege [...] no es una doctrina explícita, defendida o justificada abiertamente" (p. 167) y que en Russell "el asunto es aún más problemático" (loc. cit.), lo cual debería suscitar sospechas respecto a la tesis misma que funciona como columna vertebral del libro". (p. 189)

\section{Van Heijenoort frente a Merrill y Jaakko Hintikka}

La omisión y el comentario posterior de Tomasini son particularmente reveladores si tomamos en cuenta que en su reseña hay la presunción de conocer el trasfondo teórico sobre el cual se levantan las tesis principales de mi libro; es decir, los artículos de van Heijenoort no le son desconocidos, y, lo que es todavía más comprometedor, cuando él dice seguir, en cuanto a Wittgenstein, parcialmente a Hintikka (p. 189). Veamos por qué.

Frege solía ilustrar las diferencias entre su conceptografía y el álgebra de la lógica de Boole y Schröder apelando a la distinción que Leibniz presuntamente hacía entre una lingua characterica y un calculus ratiocinator. Con ello quería poner de relieve, entre otras cosas, que su lógica es en lo fundamental un lenguaje ideal para la expresión fiel del pensamiento, mientras que los sistemas de Boole y Schröder son meros cálculos sin aplicación alguna a la vista. No obstante, como en Leibniz, la distinción no era

\footnotetext{
${ }^{1}$ En las primeras líneas de su "Absolutismo y relativismo en lógica", van Heijenoort escribe: "Frege es quizás el lógico que más firmemente ha defendido el absolutismo en lógica. No como una tesis explícitamente enunciada y defendida por una elaborada argumentación, sino como un tema siempre presente que penetra todos sus escritos y explica muchos de sus rasgos" (van Heijenoort et al., Hacia una explicación de las entidades lógicas, Universidad Nacional Autónoma de México, México, 1984, p. 13).
} 
excluyente; es decir, la lógica fregeana como lingua characterica es también un cálculo pero mucho más que eso. ${ }^{2}$

En su clásico ensayo, van Heijenoort rebautizó la distinción LeibnizFrege resaltando el contraste que hay entre una lógica entendida como lenguaje y una lógica concebida como un cálculo; ${ }^{3}$ posteriormente, en 1984, en un trabajo sobre las distintas formas de entender el universo o dominio de la lógica, volvió a etiquetar ambas concepciones y se refirió a ellas como el absolutismo y el relativismo en lógica. ${ }^{4}$ Después Jaakko, y luego Merrill, Hintikka emplearon la primera formulación de van Heijenoort para referirse a dos concepciones del lenguaje: el lenguaje entendido como medio de expresión universal y el lenguaje visto como un cálculo. Para ambos, dicha extensión es necesaria si se quiere dar cabal cuenta de la filosofía de Wittgenstein. ${ }^{5}$

En lo personal me valgo más de la expresión "absolutismo lógico", en parte por dos motivos que están presentes en mi libro. El primero de ellos tiene que ver con los algebristas lógicos del siglo XIX, quienes a menudo se referían a sus respectivos cálculos como un lenguaje y, por este motivo, en el caso de Boole, intento exponer sus puntos de vista y examino si su posición corresponde a una concepción universalista de lógica o no (cfr. en mi libro $\S 3.3$ y $\S 3.4$ ); pero, además, porque en su discusión con Frege, Schröder retoma de manera explícita la distinción leibniciana y considera la conceptografía más como un calculus ratiocinator que como una verdadera characteristica universalis. Debo reconocer que este último punto se encuentra tratado de forma muy marginal (véanse las pp. 93 y 94) y que merece mayor atención y discusión de la que le he dado.

${ }^{2}$ En la nota 21, p. 195 de mi libro, hago notar que, hasta donde se sabe, Leibniz nunca usó la expresión lingua characterica, sino únicamente la expresión characteristica universalis, y que yo he empleado la primera expresión solamente porque es la que usó Frege para indicar que su lógica es, ante todo, un lenguaje fundamental. Tomasini se fija en el error gramatical (p. 191) que cometo al inicio de la nota, pero es incapaz de retener su contenido, y por tal motivo levanta quejas fuera de lugar con respecto a la relación entre ambas expresiones (pp. 186 y 187).

${ }^{3}$ En "Logic as Calculus and Logic as Language" escribió: "La oposición entre calculus ratiocinator y lingua characterica posee varias conexiones pero también aspectos muy distintos. La variedad de esos aspectos, la mayoría de las veces no expresados por Frege, ha de ser extraída mediante el estudio de su obra. De sus escritos emerge una imagen de la lógica, una concepción que, si bien quizá no es discutida explícitamente, sin duda alguna guía su trabajo. Para referirme a esta concepción hablaré de la universalidad de la lógica" (Synthese, vol. 17, 1967, pp. 324-330; la cita procede de la p. 324).

${ }^{4}$ En esta última etapa también distinguía ambas posturas recurriendo a las nociones escolásticas "logica magna" y "logica utens".

${ }^{5}$ Cfr. el primer capítulo de Merrill B. y Jaakko Hintikka, Investigating Wittgenstein (Basil Blackwell, Oxford, 1986, pp. 1-29), "Wittgenstein and Language as Universal Medium" (reimpreso como cap. 6 en el vol. 2 de los Selected Papers de Jaakko Hintikka, Kluwer Academic, Dordrecht, 1996, pp. 162-190). 
Ahora volvamos un momento a la reseña. En la página 190 dice: "Tenemos que retomar aquí la distinción de Jean van Heijenoort y de Hintikka, de la que abiertamente se sirve Hernández, entre la lógica como cálculo y la lógica como lenguaje."

Aquí hay dos imprecisiones que vale la pena resaltar. La primera, bastante obvia, es que Tomasini borra sin más la diferencia que hay entre hablar de la lógica como lenguaje, como lo hizo van Heijenoort, y hablar del lenguaje como medio universal, como lo hacen los Hintikka. No me detendré a especular si cuando escribe Hintikka se refiere sólo a Jaakko, o sólo a Merrill, o a ambos, pero en cambio quiero hacer notar que en la introducción de mi libro (pp. 20 y 21), enumero cuatro razones importantes por las cuales "no me sirvo" de la interpretación de los Hintikka. Y el motivo principal es bastante simple: basta describir la concepción absolutista de la lógica de Wittgenstein para explicar la filosofía del Tractatus.

Pero Tomasini no sólo evita las aclaraciones pertinentes, sino que se lanza sin más a la crítica:

gracias a Hernández nos enteramos también de que prácticamente el todo del Tractatus "puede explicarse muy bien apelando únicamente a la concepción absolutista de la lógica que éste hereda de Frege y Russell" (p. 21): esto es de un simplismo filosófico que raya en lo absurdo: ¿o sea que se llega también a la filosofía de la ciencia, a lo que nos dice sobre la ética, a la definición del número, etc., a través de meras deducciones a partir de la 'tesis absolutista' (como si, por si fuera poco, el Tractatus contuviera tesis)? iAbsurdo! (p. 188)

Aunque Tomasini dice un poco más adelante (p. 189):

no tengo reparos en relación con la adscripción a Wittgenstein de la concepción absolutista de la lógica que yo (siguiendo en esto parcialmente a Hintikka) entiendo del siguiente modo: la lógica es el medio universal, un universo del cual no hay salida. La lógica es el gran espejo de la realidad, pues sus límites fijan los límites de la factualidad, esto es, del mundo [...]. En verdad, puede sostenerse que Wittgenstein es el único filósofo que se tomó en serio la demostración fregeana de que hay cosas que no se pueden decir, sólo que generalizó el resultado de Frege concerniente a los conceptos al todo del lenguaje de un modo que para Frege habría sido impensable. Es, pues, en este sentido que la lógica es absoluta y si es esta posición la que se le adscribe al Tractatus estoy totalmente de acuerdo con el autor.

Nótese que, de nuevo, Tomasini no habla con respecto a Wittgenstein de la universalidad del lenguaje, como lo hacen los Hintikka, sino de la universalidad de la lógica. Quizá su acuerdo con "Hintikka" se restringe al empleo de la noción "medio universal"; pero no deseo discutir en esa dirección; lo que quiero resaltar es que si Tomasini rechazara atribuir a Wittgenstein 
el absolutismo en lógica, podría entenderse que mi criterio de simplicidad explicativa le resulte absurdo; sin embargo, al aceptar dicha atribución, su comentario es una reacción injustificada, pues se puede estar de acuerdo o no con el alcance de mi explicación, pero tildarla de absurda no es más que abusar del lenguaje. Por otra parte, debo reconocer que en mi libro no doy una explicación de todos los puntos que aquí señala Tomasini, aunque, como espero mostrar en cuanto a los números y la ética, una vez que se han comprendido los planteamientos básicos de la interpretación absolutista no es difícil apreciar cómo se relacionan aquellos aspectos básicos aparentemente marginales de su filosofía.

Pero antes de llegar ahí permítaseme recapitular y volver al reclamo inicial.

\section{Definiciones}

Como ya he mencionado, Tomasini lamenta la falta de "una respuesta clara y unívoca" (p. 184) sobre la concepción absolutista de la lógica, "que rece más o menos así: "El absolutismo lógico es la doctrina que sostiene que o de acuerdo con la cual...'” (p. 185). Y un poco más adelante escribe: "habría que admitir que el capítulo sobre Leibniz es instructivo, si bien adolece del defecto general del libro: las inexactitudes, la falta de definiciones (lo cual no deja de ser sorprendente al tratarse de un admirador recalcitrante de Frege), las tergiversaciones" (p. 186).

Aquí lo primero que hay que hacer es devolver el cumplido: a mí me sorprende que un admirador de Wittgenstein reclame definiciones. Los que conocen los trabajos de van Heijenoort ya mencionados saben que ahí no ofrece definición alguna del absolutismo lógico, sino que presenta, como hacen igualmente los Hintikka, "several clarificatory comments" [algunos comentarios esclarecedores]. No me arropo en el prestigio de estos investigadores para esconder mis propias faltas, sino para sugerir que la cuestión es algo que, parafraseando a Wittgenstein, se encuentra conectado con la naturaleza misma de la investigación.

Desde luego, esto no quiere decir en modo alguno que no se pueda dar una definición del absolutismo lógico, pero que semejante definición resulte adecuada y completa es otra cosa y muy cuestionable. La versión de Tomasini del absolutismo en Wittgenstein, citada anteriormente, es, como veremos un poco más adelante, insuficiente y menos clara y unívoca de lo que él mismo cree.

Ya he dicho que un rasgo importante de la concepción absolutista de la lógica es el hecho de tratarse de una doctrina no enunciada de manera categórica y que uno debe adentrarse en el estudio detallado de las obras de Frege, Russell y Wittgenstein para extraer sus características más sobresalientes. Ahora bien, para hacer explícito lo implícito resulta de poca o 
nula ayuda recurrir a las definiciones, pues es a través del comportamiento de esos sistemas lógicos como descubrimos su naturaleza. Y, por lo tanto, para ello no necesitamos definir, sino describir. En ese sentido podríamos decir, a la Wittgenstein, algo más o menos así: "Examina cómo los cuantificadores de Frege y Russell ligan sus variables, qué cantidades tan grandes de objetos pueden reemplazar sus variables; observa cómo ambos tipos de cuantificadores van más allá de la lógica de primer orden, y cómo, a pesar de sus diferencias al establecer el rango del dominio de las variables, ambos poseen un solo universo, que identifican con el universo, etcétera. Pero no pienses en una definición, isólo mira!"

La descripción de los rasgos absolutistas puede, por supuesto, variar en forma y fondo tanto como se quiera, o, si se prefiere, tanto como lo permitan nuestros escrúpulos filosóficos. ${ }^{6}$ En lo particular, en mi libro no sigo una descripción tipo Wittgenstein, sino más bien una descripción genealógica que va de Leibniz a Wittgenstein, estableciendo sus semejanzas pero también sus diferencias. Y dado que la distinción entre una lingua characterica y un calculus ratiocinator es empleada por Frege para establecer sus acuerdos y desacuerdos con respecto a otros sistemas lógicos, como los de Boole y Schröder, pero también con el de Peano, intento tomar en cuenta, tanto como me ha sido posible, el ambiente más amplio sobre el que se dan dichas rivalidades (cfr. en especial el cap. III).

Pero Tomasini no sólo tiene una idea equivocada de mi trabajo, sino también del papel que desempeñan las definiciones en Frege. Para este último, como para Russell y Wittgenstein, las definiciones son únicamente abreviaciones de expresiones más largas; esto es, simples recursos de comodidad en la expresión. ${ }^{7}$ Lo cual ciertamente no quiere decir que no posean criterios para establecer cuándo una definición debe ser adecuada (cfr. en mi libro la discusión Frege-Peano, § 3.5, y la discusión RussellPeano, § 4.6), ni que haya una enorme diferencia entre el valor de las definiciones dentro de un sistema lógico o matemático, y la pertinencia de las mismas dentro de un trabajo como el mío.

\footnotetext{
${ }^{6}$ En el caso de los Hintikka encontramos, por ejemplo, el siguiente comentario: "Since he [Frege] does not believe in the proper linguistic expressibility of such semantical relationships, he does not incorporate them in his 'official' systematic theory, but leaves them on the level of indirect informal explanations" [Dado que [Frege] no cree que tales relaciones semánticas sean propiamente expresables, no las incorpora en su teoría sistemática "oficial", sino que las introduce por medio de explicaciones informales indirectas], op. cit., p. 2.

${ }^{7}$ Cfr. Frege, Conceptografía (trad. de Hugo Padilla, UNAM, México, 1972), § 8; Russell y Whitehead, Principia mathematica (Cambridge University Press, Cambridge, 1927), vol. I, p. 11: "Practically, of course, if we introduced no definitions, our formulae would very soon become so lenghty as to be unmanageable; but theoretically, all definitions are superfluous" [En la práctica, por supuesto, si no introdujéramos definición alguna, nuestras fórmulas rápidamente se harían tan largas que resultarían inmanejables; pero teóricamente todas las definiciones son superfluas]. Y Tractatus 4.241 y 4.242 .
} 
Pero, además, hay muchas cosas en Frege, y muy importantes, que no se pueden definir y esto se debe, de nueva cuenta, a su concepción universalista de la lógica. Si la lógica es, según Frege, nuestro lenguaje más fundamental, debe ser claro que no se puede ir más allá de éste para definir sus elementos básicos. Esto es, no sólo no se puede definir qué son los conceptos (lo cual es muy distinto de decir, como afirma Tomasini, que no se puedan expresar, p. 198), tampoco podemos definir qué son los objetos (primera omisión en la versión de Tomasini citada anteriormente), ni definir qué es la verdad (segunda omisión), y lo mismo se puede decir de las leyes lógicas (tercera omisión) y de otras entidades lógicas. ${ }^{8}$ Pero volvamos de nuevo a la reseña.

\section{Absolutismo y teoría pictórica}

En dos ocasiones (pp. 185 y 189-190), Tomasini me atribuye una equivalencia entre el absolutismo lógico y la teoría pictórica del Tractatus, que, la verdad sea dicha, ignoro de dónde salió. No ofrece una sola cita mía que avale o preste el mínimo apoyo a su afirmación y el lector simplemente tiene que creerle, sobre todo si, como sostiene, mi libro se encuentra plagado de absurdos, inexactitudes, galimatías, inconsistencias, tergiversaciones e incomprensiones (en su reseña me regala cuarenta adjetivos de la misma clase).

Sin embargo, con ello lo único que Tomasini demuestra es que simplemente no ha entendido cuál es el quid de mi interpretación. Lo que afirmo con respecto al Tractatus es que la concepción absolutista se manifiesta por medio de la interpretación semántico-metafísica que Wittgenstein hace de las expresiones del cálculo lógico bivalente. Lo cual, ciertamente, incluye la llamada teoría pictórica, ya que se identifica con el tipo de expresiones que se encuentran delimitadas por las expresiones que Wittgenstein considera propiamente lógicas; es decir, la teoría pictórica refiere a aquellas expresiones que llamamos comúnmente factuales, de hecho, contingentes o sintéticas, en contraposición a las expresiones necesarias (tautológicas) y las expresiones imposibles (contradictorias), que son las que las delimitan. El cuadro 1 de la página siguiente es un esquema de la interpretación del cálculo bivalente en el Tractatus (el cual es una reelaboración de la matriz que aparece en la p. 173 de mi libro) que permite visualizar el lugar que ocupa la teoría pictórica. El meollo de la interpretación absolutista del

${ }^{8}$ A diferencia de las nociones de objeto y concepto, las cuales han de entenderse por medio de alusiones o, como dicen los Hintikka ya mencionados, por medio de explicaciones indirectas e informales, el significado de la noción de verdad lo captamos por medio de las leyes lógicas, que en Der Gedanke denomina leyes del ser verdad, mientras que estas últimas las entendemos a través de su uso y, por consiguiente, por sus diferencias con las leyes naturales. Cfr. mi libro, § 2.4 . 


\begin{tabular}{|c|c|c|}
\hline Necesidad & Posibilidad & Imposibilidad \\
\hline$\downarrow$ & $\downarrow$ & $\downarrow$ \\
\hline Tautología & Proposición & Contradicción \\
\hline$\downarrow$ & $\downarrow$ & $\downarrow$ \\
\hline $\begin{array}{c}\text { Expresiones } \\
\text { sin sentido (sinnlos) }\end{array}$ & $\begin{array}{c}\text { Expresiones } \\
\text { con sentido (Sinn) }\end{array}$ & $\begin{array}{c}\text { Expresiones } \\
\text { sin sentido(sinnlos) }\end{array}$ \\
\hline$\downarrow$ & $\downarrow$ & $\downarrow$ \\
\hline$\overline{\mathrm{V}}$ & $\mathrm{V}$ & $\bar{F}$ \\
\hline$\overline{\mathrm{V}}$ & $\bar{F}$ & $\bar{F}$ \\
\hline$\uparrow$ & $\uparrow$ & $\uparrow$ \\
\hline Expresiones tipo $\alpha$ & Expresiones tipo $\beta$ & Expresiones tipo $\alpha$ \\
\hline$\uparrow$ & $\uparrow$ & $\uparrow$ \\
\hline $\begin{array}{c}\text { Límite del lenguaje } y \\
\text { del mundo }\end{array}$ & $\begin{array}{l}\text { Teoría figurativa o } \\
\text { pictórica del lenguaje }\end{array}$ & $\begin{array}{c}\text { Límite del lenguaje } \\
\text { y del mundo }\end{array}$ \\
\hline
\end{tabular}

CUADRO 1. Estructura básica de la interpretación semántico-metafísica del cálculo bivalente en el Tractatus

Tractatus consiste, por lo tanto, en que la estructura del cálculo bivalente se identifica con la estructura del lenguaje y del mundo. Y es de esta manera que, interpretadas de forma claramente absolutista, las expresiones lógicas (las expresiones tipo $\alpha$ ) marcan los límites del pensamiento, del mundo y del lenguaje, mientras que las expresiones factuales (o tipo $\beta$ ), encerradas, o, para usar un término de moda, encapsuladas en la lógica, establecen un isomorfismo entre lenguaje y realidad. En consecuencia, cuando considera mi interpretación, Tomasini confunde una parte con el todo.

No obstante, aunque ésta es la verdadera dimensión de mi interpretación, de todos modos Tomasini tampoco la aceptaría:

¿Qué conexión hay entre el absolutismo lógico y la Teoría Pictórica? Se trata de dos cuestiones lógicamente independientes [...]. Lo que guía a Wittgenstein en su investigación es obviamente el lenguaje lógico, esto es, el simbolismo russelliano. Eso lo lleva a decir, entre muchas otras cosas, que las proposiciones del lenguaje natural son retratos (Bilden, pictures), puesto que expresiones como ' $\phi x$ ' son retratos. Pero a partir de esa investigación que versa sobre signos, Wittgenstein elabora o construye una concepción "absolutista" de la lógica que es independiente de su estudio del simbolismo. Se sigue que su concepción de la lógica es independiente de la Teoría Pictórica. (p. 190)

Hay varias formas de desacreditar esta opinión, empezando por su falta de claridad: ¿es efectivamente ' $\phi x$ ' una proposición del lenguaje natural?, y si es así, ¿qué hecho retrata?, ¿difieren ' $\phi x$ ' y ' $\phi x \rightarrow \phi x$ ' sólo en notación 
o muestran una diferencia más fundamental? Pero basta notar que su argumento final es tan fascinante como contundente, ya que la conclusión se limita a repetir la segunda premisa, y, curiosamente, es la segunda premisa lo que se busca probar: $x$ se construye a partir de $y$ y, a la vez, $x$ es independiente lógicamente de $y$. Luego, $x$ es independiente lógicamente de $y$. En fin, sea como sea, lo único que queda claro es que el absolutismo que Tomasini le atribuye a Wittgenstein es bastante nominal y secundario. Así que será mejor regresar a mi interpretación.

En el Tractatus hay además un tercer tipo de expresiones (digamos de tipo $\varepsilon$ ), que se genera como el complemento de las expresiones $\alpha$ y $\beta$. Tales expresiones son las que Wittgenstein llama absurdos (unsinning), y en símbolos podemos describirlas de la siguiente manera: $\varepsilon=\langle\alpha-\beta\rangle$. Para variar, volvamos ahora a uno de los muchos absurdos que Tomasini me imputa. En 6.42, Wittgenstein señala que no puede haber proposiciones éticas, y, que, por consiguiente, la ética no puede expresarse. Y es justo en este sentido que se dice que la ética es trascendente (6.421). Esto es así porque en la interpretación absolutista del Tractatus sólo cuentan como lenguaje aquellas expresiones que pueden ser sujetas a valoración de verdad y, como es de sobra sabido, a un enunciado ético no es posible asignarle valores de verdad. Un imperativo como "no robarás" no es algo al que tenga sentido atribuirle un valor de verdad, y, por supuesto, hay muchas otras expresiones que no son objeto de valoración epistémica. Sin embargo, Wittgenstein es un absolutista radical, de modo que cuando intentamos expresar un enunciado ético, lo único que conseguimos, según él, es un mero absurdo; esto es, una expresión tipo $\varepsilon$. Además, Wittgenstein tenía conocimiento de experiencias que cuadraban perfectamente con su interpretación y es así como en 6.521 resuelve el asunto de la siguiente manera: "La solución del problema de la vida está en la desaparición de este problema. (¿No es ésta la razón de que los hombres que han llegado a ver claro el sentido de la vida, después de mucho dudar, no sepan decir en qué consiste este sentido?)" Sin duda, uno de los mayores atractivos del Tractatus consiste precisamente en esto: en haber colocado la experiencia mística sobre un fundamento lógico.

Ahora recapitulemos: Tomasini me imputa, erróneamente, una equivalencia entre absolutismo lógico y teoría figurativa del lenguaje. Pero también me atribuye una equivalencia entre absolutismo y logicismo, con el fin de construir un argumento con una conclusión inadmisible, que va así:

1. El absolutismo lógico = teoría pictórica del lenguaje.

2. El absolutismo lógico = logicismo.

Por lo tanto, logicismo = teoría pictórica del lenguaje. 
Como ya he demostrado la falsedad de la primera premisa, esto es suficiente para echar por tierra este argumento. Sin embargo, ¿qué hay sobre la segunda premisa? Su fundamento parece encontrarse en la sexta cita (p. 184), que presenta así:

f) "la nueva versión russelliana del programa absolutista, conocida más comúnmente como logicismo" (loc. cit.).

La lectura que Tomasini hace de este pasaje es tan pobre que ofende, y para conjurarla bastará echar mano de un poco de dialéctica socrática:

A: Ya se ha dicho que el absolutismo lógico es una concepción acerca de la naturaleza de la lógica, ¿no es así?

B: En efecto.

A: ¿Es, pues, el logicismo una concepción sobre la lógica?

B: De ningún modo.

A: Entonces, ¿puede ser lo mismo o equivalente al absolutismo?

B: Desde luego, no.

A: Pero ¿acaso no se ha dicho también que la lógica de los logicistas presupone una concepción que van Heijenoort y otros llaman absolutista o universalista?

B: Sin duda.

A: Entonces, ¿cómo puedes parafrasear f) correctamente; es decir, sin establecer una equivalencia entre logicismo y absolutismo?

B: Del siguiente modo: "La concepción absolutista de Russell la conocemos a través de su logicismo".

\section{A: Perfecto.}

Llama la atención, no obstante, que cuando Tomasini nos describe cómo entiende el logicismo (p. 185), de alguna manera admite justamente lo que acabamos de decir, aunque, para sorpresa de todos, según él no hay nada en el logicismo que presuponga una concepción de la lógica. Pero vayamos por partes.

\section{Logicismo}

Como he señalado anteriormente, Tomasini construye un argumento sobre la base de dos premisas apócrifas con la intención de desacreditar mi interpretación del absolutismo lógico. Muy bien, ¿y ahora? Luego se esfuerza en demostrar que mis intenciones son simplemente absurdas y, por lo tanto, inadmisibles. Y agrega: "Recuérdese que al primer enemigo decidido del logicismo lo encontramos en el Tractatus mismo" (p. 185). Entonces intento recordar, pero sólo me vienen a la memoria Poincaré y Boutroux redactando acalorados escritos contra el primer logicismo de Russell, justo 
cuando Wittgenstein rondaba los quince años... luego recuerdo a Couturat defendiendo por escrito la causa logicista, sin mucho éxito, contra Poincaré, mientras que ante Borél y Lebesgue lo hacía sotto voce. Entonces me pregunto, ¿qué quiere decir aquí exactamente Tomasini? La verdad, lo ignoro.

Todo el apoyo que cita a su favor es la primera frase de la observación 6.031 del Tractatus: "la teoría de clases es superflua en matemáticas". Confieso lo difícil que me resulta entender esta afirmación como una negación del logicismo, aunque también admito que semejante observación ha dado lugar a una interpretación muy extendida. Y los motivos parecen ser sumamente transparentes ya que, se razona, para definir lógicamente los números naturales necesitamos las clases, pero como, al mismo tiempo, Wittgenstein niega que en realidad sea necesario recurrir a tales entidades, entonces, se concluye, no es posible definir lógicamente los números naturales y, por lo tanto, el logicismo debe desecharse.

Sin embargo, no es muy difícil darse cuenta de que dicha interpretación resulta insostenible cuando se examina la cuestión con detalle, lo cual ciertamente no suele hacerse (basta echar un vistazo a la abultada literatura que se ha escrito sobre el Tractatus para percatarse de que el asunto ha sido soslayado, con notables y contadas excepciones). Ahora bien, justo antes de la cita que da pie a la mencionada interpretación aparece la definición de Wittgenstein de los números naturales positivos, y lo mínimo que puede hacerse al respecto es preguntarse: ¿es posible definir lógicamente los números enteros sin recurrir a las clases?, ¿sigue siendo, después de todo, la definición de Wittgenstein una definición lógica?

De acuerdo con la interpretación a la que Tomasini se adhiere, la respuesta a ambas preguntas debe ser negativa. Entonces la cuestión siguiente es averiguar qué tipo de definición se ofrece allí. Pero de aquí en adelante las opciones no son en absoluto prometedoras, ya que se tendrá que optar o bien por una definición en términos de proposiciones factuales (o pictóricas, si se prefiere), lo cual significaría ejercer una violencia extrema sobre las tesis principales del Tractatus, o bien, elaborarla por medio de expresiones absurdas (las expresiones tipo $\varepsilon$ de nuestro cuadro), lo cual representaría reconocer que no se ha dado definición alguna. Desde luego, cualquier otra alternativa, que no sea la definición lógica, saldrá, irremediablemente, de los tres tipos de expresiones del Tractatus y tendrá que desecharse.

Pero supongamos ahora que el defensor de la interpretación antilogicista acepta una respuesta negativa a la primera pregunta y una respuesta afirmativa a la segunda (debe haber quedado claro que la posibilidad inversa conduce también al atolladero antes mencionado). Entonces tendrá que admitir que hay una clara incoherencia entre la definición y la observación citada (6.031), lo cual resulta de igual modo inaceptable. Por el contrario, 
si se acepta una respuesta afirmativa a ambas preguntas, la interpretación perderá toda su fuerza y quedará reducida a nada.

Pero dejemos de lado lo anterior y pasemos, aunque sea de manera informal y escueta, a la definición misma. Lo que hace Wittgenstein es presentar una fórmula general del número (6.03), que se obtiene a partir de la fórmula general de la operación (6.01) que se encuentra en la base de la forma general de la proposición (6), la cual, a su vez, da origen a la matriz incluida en el cuadro 1 . Pero para entender correctamente estas fórmulas generales basta pensar no en la teoría pictórica, sino en la semántica del cálculo lógico. En efecto, lo que hay en común en la forma general de la función de verdad y en la fórmula general del número es la noción de operación iterativa, y es a causa de este desarrollo técnico por lo cual Wittgenstein sostiene que la matemática es un método lógico (6.2 y 6.34). En clave absolutista, esto significa que "La lógica del mundo, que en la lógica se muestra en las tautologías, en la matemática se muestra en ecuaciones" (6.22). En consecuencia, su concepción puede entenderse si bien no como un logicismo a la Frege-Russell, sí como una especie de logicismo. ${ }^{9}$

No está de más añadir que Russell encontraba insuficiente el método de Wittgenstein, pero no ajeno o incompatible con los principios del logicismo. Todo lo contrario, como lo demuestra el siguiente comentario:

Hay algunos aspectos en los cuales, me parece, la teoría de Wittgenstein todavía requiere mayor desarrollo técnico. Me refiero en particular a su teoría del número (6.02 y ss.), la cual, tal como está, sólo es capaz de ocuparse de los números finitos. Ninguna lógica puede considerarse adecuada hasta que ha mostrado ser capaz de tratar con números transfinitos. No creo que haya nada en el sistema de Wittgenstein que lo imposibilite a llenar esta laguna. ${ }^{10}$

Pero también Tomasini olvida o ignora que Wittgenstein no fue el primero en pensar en una alternativa a las clases, pues, ahora sí, recuérdese que el 24 de mayo de 1903, Russell escribe a Frege: "creo haber descubierto

\footnotetext{
${ }^{9}$ Pasquale Frascolla, quien es de los pocos que se han ocupado con detenimiento en la cuestión, afirma: "the label 'no-classes logicism' turn out to tally with the Tractatus view of aritmethic" [la etiqueta 'logicismo sin clases' parece ajustarse bien a la concepción de la aritmética del Tractatus] ("The Tractatus System of Arithmetic", Synthese, vol. 112, 1997, p. 354; y más adelante p. 361).

10 “There are some respects, in which, as it seems to me, Mr. Wittgenstein's theory stands in need of greater technical development. This applies in particular to his theory of number (6.02 ff.) which, as it stands, is only capable of dealing with finite numbers. No logic can be considered adequate until it has been shown to be capable of dealing with transfinite numbers. I do not think there is anything in Mr. Wittgenstein's system to make it impossible for him to fill this lacuna" (introducción al Tractatus, p. xx; cito a partir de la edición de Pears and McGuinness, Londres, Routledge and Kegan Paul, 1961).
} 
que las clases son por completo superfluas". ${ }^{11}$ Este déjà vu, que debe resultar sumamente embarazoso para la interpretación de Tomasini, no puede entenderse como una claudicación del logicismo. Pero ¿qué mueve aquí a Russell, y después a Wittgenstein, a rechazar las clases? Todo mundo sabe que la paradoja de Russell echa sus raíces en la teoría de clases, y que para el logicismo era prioridad deshacerse de ella si quería concluir con éxito la realización del programa que se proponía. Russell descubrió la paradoja en junio de 1901, pero tardó más de un año en informarle a Frege su fatal descubrimiento. El 20 de octubre de 1902, este último escribe a Russell ofreciéndole una posible solución: reemplazar el axioma o ley $\mathrm{V}$ por un nuevo axioma $V^{\prime}$. Russell se muestra entonces escéptico ante dicha alternativa y busca otras posibles soluciones. La observación de Russell citada anteriormente apunta hacia una de ellas, aunque su existencia fue efímera ya que sería sustituida definitivamente por la teoría de los tipos lógicos (lo cual devolvía a las clases su estatus legítimo). Ahora bien, la observación similar de Wittgenstein debe entenderse, en esta perspectiva histórica, como un intento renovado por evitar las clases, pero sin que esto suponga una renuncia al logicismo. En consecuencia, señalar, como lo hace Tomasini, que "el Tractatus es el primer enemigo decidido del logicismo" es algo más que una simple exageración.

Pero eso no es todo, la interpretación de Tomasini es incompatible con la caracterización ingenua que nos presenta del logicismo, pues, según él,

es simplemente la tesis de la reducción de las matemáticas a la lógica, y, en sí mismo, no contiene o acarrea ninguna concepción particular de la lógica. En efecto, se puede ser un logicista, en el sentido de pensar que todos los conceptos matemáticos pueden ser definidos por medio de conceptos lógicos y que todas las verdades matemáticas pueden ser traducidas a verdades lógicas, sin por ello tener necesariamente que defender una posición absolutista de la lógica. Se trataría sencillamente de la reducción de un simbolismo a otro. ${ }^{12}$

De acuerdo con esta forma de entender las cosas, si Brouwer y Hilbert no fueron logicistas, fue simplemente porque no les dio la gana. Pero nada de eso; para ser logicista se necesitaba un tipo especial de lógica y ésta encierra ya una concepción particular sobre la misma. Cavaillès gustaba plantear la cuestión de la siguiente manera: “¿Es posible hablar todavía de un fundamento por la vía de la reducción lógica? Sólo a condición de extender desmesuradamente la lógica."13 En efecto, en el caso de Frege y Russell, esa extensión desproporcionada los lleva más allá de la lógica de

${ }^{11}$ Frege, Philosophical and Mathematical Correspondence, edición abreviada de B. McGuinness, trad. al inglés de Hans Kaal, Oxford, Blackwell, p. 158.

12 P. 185, las cursivas son mías.

${ }^{13}$ Método axiomático y formalismo, traducción de Carlos Álvarez y Santiago Ramírez, UNAM, México, 1992, p. 62. 
primer orden, de tal forma que su uso de los cuantificadores presupone ya el absolutismo. Pero incluso Ramsey, quien cuenta con el mérito de haberse desecho de la teoría ramificada de los tipos lógicos, no escapó a las pretensiones omniabarcadoras de la lógica, y es por ello que Carnap no dudó en tildar su propuesta de "theological mathematics".

Pero esto es lo de menos, porque, por un lado, es obvio que si la caracterización de Tomasini fuese correcta, entonces nos debería una explicación de los motivos que llevan supuestamente a Wittgenstein a convertirse en el enemigo principal de un logicismo filosóficamente inofensivo. Y, por otro lado, es igualmente obvio que Tomasini sacrifica también su propia interpretación antilogicista, ya que según él mismo no hay ninguna razón para que las definiciones lógicas tengan que limitarse al uso de las clases.

\section{Russell}

Y ya que estamos hablando de Russell, será conveniente dedicarle unas cuantas líneas y comentar algunos de los reparos que Tomasini me ha hecho al respecto. Lo primero que merece la pena resaltar es que él no está muy seguro si Russell es a fin de cuentas un absolutista o no (p. 184), ya que:

una de las consecuencias más decisivas del absolutismo es justamente la imposibilidad de un metalenguaje y, por ende, la imposibilidad de enunciar en el lenguaje los rasgos lógicos de este último. En verdad, en hacer tal cosa consiste la paradoja mayúscula del Tractatus. Pero es precisamente en contra de esta posición de Wittgenstein (y de Frege) que Russell se subleva y propone la idea de una jerarquía de lenguajes. Independientemente de quién tenga razón, lo que queda claro es que para Russell no había obstáculo alguno para describir los rasgos formales del lenguaje (un problema que él señala desde el "Apéndice" incluido en Los principios de las matemáticas, dedicado a la filosofía de Frege, cuya posición rechaza) y, por lo tanto, hay un sentido en el que él no acepta lo que sería la principal consecuencia del absolutismo. (pp. 185 y 186)

Los comentarios de Tomasini son tan crípticos que dan pie a varios malentendidos si no se pone de manifiesto que está mezclando dos momentos bastante distintos bajo una misma perspectiva. Pero no voy a abusar de ello para lanzarle cuestionamientos injustificados, sino que intentaré dar una respuesta adecuada a sus dudas. En primer lugar, debe aclararse que al inicio de su comentario se refiere implícitamente a la parte final de la introducción al Tractatus, en la cual Russell manifiesta su insatisfacción con la condición paradójica de la propuesta de Wittgenstein. Que Russell proponga una jerarquía de lenguajes como plataforma de una revuelta, como afirma Tomasini, es hasta cierto punto cuestionable, ya que, como se dice ahí mismo, la posición del Tractatus sugiere ya esa jerarquía como una 
posible solución y Russell simplemente la explora: "Lo que provoca duda es el hecho de que, después de todo, Wittgenstein se las arregla para hablar acerca de una gran cantidad de cosas que no se pueden expresar, lo cual sugiere al lector escéptico la posibilidad de que exista alguna escapatoria mediante una jerarquía de lenguajes o por medio de alguna otra salida."14

Pero si a fin de cuentas Russell propone o no una jerarquía de lenguajes no es un asunto que nos lleve a determinar si es o no un absolutista en cuestiones de lógica. Dicho de otra manera, lo que le perturba es la consecuencia indeseable a la que se llega a partir de la versión absolutista de la lógica en el Tractatus. Es, pues, la manera paradójica como se resuelven las cuestiones en el Tractatus lo que lo deja intelectualmente insatisfecho; y si efectivamente propone una jerarquía de lenguajes, sólo demuestra que no era consciente de que dicha posibilidad se encuentra cancelada desde adentro.

En tal condición reside el problema crucial de la concepción universalista. En efecto, el absolutismo impone restricciones severas sobre el modo de empleo del simbolismo, de tal suerte que tarde o temprano terminan por violentarse las prohibiciones autoimpuestas, ya sea de manera inconsciente, como en el caso de Russell, o bien, con plena conciencia de sus consecuencias, como ocurre con Wittgenstein. En el caso de Frege, la paradoja no salta a la vista de manera tan evidente como ocurre en Wittgenstein, pero no por ello deja de estar ahí; como señalo en mi libro (cfr. $§ 2.4$ y $\S 2.6$ ), una de las mayores ironías que uno encuentra en Frege consiste en haber creado un lenguaje lógico con el propósito de superar las deficiencias que posee el lenguaje natural cuando pretende dar cuenta de las relaciones lógicas que se dan entre los pensamientos, y que, al mismo tiempo, para hacer inteligibles distinciones lógicas fundamentales, como la distinción entre objeto y concepto y la que hay entre la proposición y su valor de verdad, haya tenido necesidad de recurrir al lenguaje natural, apelando en el primer caso a distinciones gramaticales — como el uso de los artículos definidos e indefinidos- y, en el segundo caso, a la relación que guarda un nombre propio con el objeto al que designa.

Por otra parte, una razón importante por la cual Russell no era muy consciente de su absolutismo es que, a diferencia de Frege, se da a la tarea de desarrollar los fundamentos de las matemáticas sin llegar a una solución satisfactoria sobre los compromisos ontológicos adquiridos por el aparato lógico. La razón que nos da, por ejemplo, en Principia, es que aún no posee métodos poderosos para vérselas con los problemas de la filosofía de la lógica: "Nuestro sistema se construye a partir de 'proposiciones atómicas'.

14 "What causes hesitation is the fact that, after all, Mr. Wittgenstein manages to say a good deal about what cannot be said, thus suggesting to the sceptical reader that possibly there may be some loophole through a hierarchy of languages, or by some other exit" (Russell, op. cit., p. xxi). 
Las aceptamos como algo dado porque los problemas a que dan pie pertenecen a la parte filosófica de la lógica, y no pueden someterse (al menos hasta ahora) a un tratamiento matemático." 15

No obstante, como observa van Heijenoort, ${ }^{16}$ es sumamente sintomático que los ejemplos que Russell presenta de semejantes proposiciones sean expresiones tales como "esto es rojo", o "esto es primero que aquello", que, en principio, nada tienen que ver con los fundamentos de las matemáticas. Y si tales proposiciones elementales remiten a lo que Wittgenstein llama figuras o retratos, en cuanto que la especificación de todas ellas nos da una descripción completa del mundo (4.26), no se trata de una mera casualidad, sino de un indicio claro de que éste hereda de Russell todas aquellas cuestiones concernientes a la filosofía de la lógica que, debido supuestamente a la disminución de sus fuerzas intelectuales, sentía que ya no era capaz de contestar.

Y si Wittgenstein se ve obligado hablar de hechos atómicos, no es más que consecuencia de la manera en que se construye el cálculo a partir de la combinatoria de los posibles valores de verdad de las proposiciones elementales. De hecho, que la teoría pictórica se remita directamente a las proposiciones elementales es una clara muestra de que se encuentra encerrada o encapsulada en la lógica absolutista, y que, por consiguiente, no puede ser, como afirma Tomasini, "lógicamente" independiente de ella.

Por otra parte, lo que Russell hace en el apéndice de los Principles es simplemente discutir algunos rasgos de la conceptografía y su aplicación a la aritmética con los cuales no está de acuerdo (y ahora sabemos también que en muchos de esos puntos no tenía aún una idea clara de la posición de Frege). Es decir, compara la distinción sentido-referencia con la suya propia, comenta si Frege tiene una concepción más intensional que extensional de las clases, etcétera; pero se trata siempre de objeciones dentro del mismo programa y tradición, y no, como afirma Tomasini, de un rechazo externo, ajeno a los presupuestos y principios absolutistas. ${ }^{17}$

15 "Our system begins with 'atomic propositions'. We accept these as a datum, because the problems wich arise concerning them belong to the philosophical part of logic, and are not amenable (at any rate at present) to mathematical treatment" (Russell y Whitehead, 1925, p. xv). Desde luego, ya que estas líneas pertenecen a la segunda edición de Principia, también indican que, para Russell, la propuesta del Tractatus sobre la naturaleza de las proposiciones atómicas no era la respuesta definitiva a la cuestión.

16 "Système et métasystème chez Russell", en Logic Colloquium '85, ed. The Paris Logic Group, North-Holland, Amsterdam, 1987, p. 111.

${ }^{17}$ Las primeras líneas del apéndice bastan para corroborar lo que digo: "In what follows, I shall try briefly to expound Frege's theories on the most important points, and to explain my grounds for differing where I do differ. But the points of disagreement are very few and slight compared to those of agreement" [En lo que sigue trataré de exponer brevemente las teorías de Frege en sus puntos más importantes, y explicaré los motivos que tengo para diferir de él allí donde difiero, aunque los desacuerdos son tan pocos e insignificantes en comparación 
Entender esto último es de suma importancia si se quiere establecer con precisión el vínculo que hay entre Frege, Russell y Wittgenstein. Pero Tomasini tiende a malinterpretar precisamente esta clase de distinciones y me atribuye filias y fobias que no son más que producto de sus propias proyecciones. No tengo ningún motivo para admirar menos a Wittgenstein y a Russell que a Frege, ni pienso que al relacionar a Wittgenstein con la characteristica universalis de Leibniz le reste originalidad o mérito alguno, sino todo lo contrario, con ello podemos comprender mejor su pensamiento. Pues, como dice Aristóteles, "de unos hemos recibido ciertas opiniones y otros fueron causa de que surgieran aquéllos". ${ }^{18}$ No es ésta, desde luego, la opinión de Tomasini: "el Tractatus, nos informa, no es más que el 'eslabón final, y no el principio, de una larga cadena intelectual' (ibid.): en otras palabras, no tiene los méritos que usualmente se le atribuyen" (p. 188).

Sin embargo, no lo culpo por pensar así, pues lo único que hace es ceñirse a la tendencia, muy extendida, de separar a Wittgenstein de Russell y Frege, exagerando o malinterpretando sus divergencias y, al mismo tiempo, restando importancia o minimizando sus complicidades y acuerdos. Se trata de una práctica "exegética" que se ha convertido en toda una tradición y goza de cierta autoridad. Al respecto me viene a la mente una reseña sobre la correspondencia Wittgenstein-Ogden, en la cual Erik Stenius, en su afán de alejar a Wittgenstein de Russell no duda lo más mínimo en corregirlo: "En una carta a Russell de 1919 se expresa aun más categóricamente: 'Creo que finalmente he resuelto nuestro problema' (Protot., p. 7). No obstante, había dicho lo mismo en 1915, en sus notas dictadas a Moore. (Nótese el uso injustificado de 'nuestro' en estas afirmaciones)." ¿No compartían Russell y Wittgenstein los mismos problemas sobre los fundamentos de la lógica? Mi convicción, manifiesta a lo largo del libro, es un rotundo sí, y en las consecuencias de esta toma de partido descansa la mayor parte de mis divergencias con mucho de lo que se ha escrito sobre Wittgenstein. Hasta qué punto merece crédito tal postura es algo que se ha de ponderar comparando con detalle y objetividad cada una de las explicaciones, y no por el número de adeptos que se inclinan por una u otra interpretación.

\section{Para terminar}

Más adelante (p. 187), Tomasini vuelve a mostrarse fuera de lugar al reprocharme "un mínimo de discusión" en torno a lo que él denomina "la con aquello en lo que coincidimos] (Bertrand Russell, Principles of Mathematics, Cambridge University Press, Cambridge, 1903, § 475).

${ }^{18}$ Metafísica, II, 15.

19 "In a letter to Russell in 1919 he expresses himself still more categorically: 'I believe I've solved our problem finally' (Protot., p. 7). However, he said the same thing in 1915 of his notes dictated to Moore. (Note the unjustified use of 'our' in these statements)" ("Wittgenstein and Ogden”, Acta Philosophica Fennica, vol. 45, 1989, p. 261). 
muy interesante discusión respecto a si toda verdad necesaria se explica en última instancia en términos de forma lógica o no". Pero, de nuevo, lo que digo en $\S 2.3$ (conceptografía y logicismo) no tiene nada que ver con esa cuestión, pues lo que me interesa resaltar ahí es, por un lado, que la aplicación de la conceptografía (esto es, el sistema lógico de Frege) le permite saber si el dominio de aplicación es analítico o no (esto es, si se deja expresar y derivar en términos estrictamente lógicos o no); y, por otro lado, que siendo la lógica el canon de lo analítico, es un error afirmar que para Frege la lógica sea analítica.

Pero Tomasini se va por la tangente y se pregunta: “¿deja la lógica de ser analítica porque sea 'el canon de lo analítico', signifique esto lo que signifique? ¿O sea que porque la lógica es el canon de lo analítico, entonces ' $\sim p \rightarrow p$ ' no es analítico?" (p. 188). Desde luego, ambas preguntas carecen de sentido y muestran claramente la falta de cuidado que pone en lo que reseña, ya que si para Frege la lógica es el canon de lo analítico (y esto sólo quiere decir aquí que 'analítico' significa "definible en términos lógicos”), preguntarse si la lógica es analítica es preguntarse si la lógica misma es definible en términos lógicos. Es evidente que el error cometido es un simple anacronismo, ya que intenta introducir una problemática posterior y ajena a Frege. Dicho de otra forma, Tomasini confunde la noción fregeana de analítico con la discusión posterior, propia del siglo xx, de si lo analítico debe entenderse como expresión verdadera o falsa "en función de la forma lógica", o bien, "por medio del significado de los términos involucrados", etcétera.

Recibido el 2 de diciembre de 2002; revisado el 9 de julio de 2003; aceptado el 12 de agosto de 2003 